\title{
Erratum to: "Comparison of ACUITY, CRUSADE, and GRACE Risk Scales for Predicting Clinical Outcomes in Patients Treated with Dual-Antiplatelet Therapy"
}

\author{
Sun Young Choi ${ }^{1}$ Moo Hyun Kim ${ }^{2}$ Victor Serebruany ${ }^{3}$ \\ ${ }^{1}$ Department of Biomedical Laboratory Science, Daegu Health \\ College, Daegu, Republic of Korea \\ 2 Department of Cardiology, College of Medicine, Dong-A University, \\ Busan, Republic of Korea \\ ${ }^{3}$ HeartDrug ${ }^{\text {TM }}$ Research Laboratories, Johns Hopkins University, \\ Towson, Maryland, United States \\ TH Open 2019;3:e36.
}

\section{ERRATUM}

In the Original Article by Choi et al. "Comparison of ACUITY, CRUSADE, and GRACE Risk Scales for Predicting Clinical Outcomes in Patients Treated with Dual-Antiplatelet Therapy" (TH Open 2018; 02(04): e399-e406) it has come to the authors attention that the wrong corresponding author was listed. The amended corresponding author address is:

Address for correspondence

Prof. Moo Hyun Kim

Department of Cardiology

College of Medicine

Dong-A University

Busan

Republic of Korea

(e-mail: KimMH@dau.ac.kr) 